Ilchmann, Achim ; Weirig, Marie-France ; Mareels, Iven M. Y. :

Modelling and adaptive control of biochemical processes

Aus:

Nonlinear control systems design 1998 : a proceedings volume from the 4th IFAC Symposium, Enschede, The Netherlands, 1 - 3 July 1998 [4th IFAC Symposium on Nonlinear Control Systems Design 1998]. Ed. by H. J. C. Huijberts ... - Oxford : Pergamon Press, 1998, ISBN 0-08-043049-X, Vol. 1, S. 447-452 


\title{
MODELLING AND ADAPTIVE CONTROL OF BIOCHEMICAL PROCESSES
}

\author{
Achim Ilchmann ${ }^{*, 1}$ Marie-France Weirig ${ }^{* *, 1}$ \\ Iven M.Y. Mareels ${ }^{* * *}$
}

\author{
* Department of Mathematics and Centre for Systems and \\ Control Engg., University of Exeter, Exeter EX\&4QE, U.K., \\ ilchmann@maths . exeter . ac . uk \\ ${ }^{* *}$ GSF-Institute of Biomathematics and Biometry, D-85758 \\ Neuherberg, FRG, weirig@gsf.de \\ ${ }^{* * *}$ Dept. of Electrical and Electronic Engg., The University of \\ Melbourne, Parkville VIC 3052, Australia, \\ i.mareels@unimelb.edu.au
}

\begin{abstract}
For a general dynamic biochemical process described by an ordinary differential equation, we introduce the concept of non-cyclic processes. This replaces the conservation of mass assumption and is sufficient to guarantee invariance of the positive orthant and boundedness of the trajectories. Non-cyclic processes are characterized in terms of the stoichiometric matrix and an algorithm is presented which decides in finitely many steps whether a process is non-cyclic or not. The concept of $\lambda$-tracking is applied to an example to regulate some external substrate concentration by the feedrate in the presence of noise corrupting the output and in the presence of input constraints. Copyright $(1998$ IFAC
\end{abstract}

Keywords: Modelling, bioprocesses, adaptive control

\section{INTRODUCTION}

\section{Nomenclature}

$\mathbb{R}_{+}$set of non-negative real numbers

$\mathbb{R}_{+}^{*}$ set of positive real numbers

In our setup, a biotechnological process consists of a set of $M$ reactions $\varphi_{1}, \ldots, \varphi_{M}$ involving $N$ components $\xi_{1}, \ldots, \xi_{N}$. The latter denote the state variables, i.e. the concentrations in the liquid phase of the reactor. Such a process is commonly specified by the following reaction scheme for the $j^{\text {th }}$ reaction:

$$
\sum_{i \in L_{j}} c_{i j} \cdot \xi_{i} \longrightarrow \sum_{i \in R_{j}} c_{i j} \cdot \xi_{i}, \quad j=1, \ldots, M .(1)
$$

\footnotetext{
1 Supported in part by the University of Exeter Research
} Fund
Here

$$
L_{j} \subseteq\{1, \ldots, N\}, \quad L_{j} \neq \emptyset
$$

denotes the set of indices of the components $\xi_{i}$ which are the reactants of the $j$-th reaction,

$$
R_{j} \subseteq\{1, \ldots, N\}, \quad R_{j} \neq \emptyset
$$

is the set of indices of the components $\xi_{i}$ which are the reaction products of the $j$-th reaction.

The quantities of each component involved in the reaction are specified by the nonnegative stoichiometric coefficients $c_{i j}$, sometimes also called yield coefficients. The rate of consumption of the reactants, which is equal to the rate of formation of the reaction products, is called the reaction rate and denoted by $\varphi_{j}$.

We assume that the dynamics of the process (1) can be described by an ordinary differential equation: 


$$
\dot{\xi}(t)=K \varphi(\xi(t))-D(t) \xi(t)-Q \xi(t)+F(t),
$$

with initial condition $\xi(0) \in\left(\mathbb{R}_{+}^{*}\right)^{N}$ and concentration vector

$$
\xi(t)=\left(\xi_{1}(t), \ldots, \xi_{N}(t)\right)^{T} \in \mathbb{R}_{+}^{N},
$$

vector of reaction rates

$$
\varphi(\xi)=\left(\varphi_{1}(\xi), \ldots, \varphi_{M}(\xi)\right)^{T} \in \mathbb{R}_{+}^{M}
$$

with locally Lipschitz $\varphi_{j}: \mathbb{R}_{+}^{N} \rightarrow \mathbb{R}_{+}$, the matrix of stoichiometric coefficients

$$
K=\left[K_{1}, \ldots, K_{M}\right]=\left(k_{i j}\right) \in \mathbb{R}^{N \times M},
$$

locally integrable dilution rate

$$
D(\cdot): \mathbb{R}_{+} \longrightarrow[\underline{D}, \bar{D}], \quad 0<\underline{D} \leq \bar{D},
$$

the matrix of proportional gazeous outflow rates

$$
Q=\operatorname{diag}\left\{q_{1}, \ldots, q_{N}\right\}, \quad q_{1}, \ldots, q_{N} \geq 0,
$$

and the vector of feed rates

$$
F(t)=\left(F_{1}(t), \ldots, F_{N}(t)\right)^{T} \in \mathbb{R}_{+}^{N}
$$

with locally integrable $F_{i}(\cdot): \mathbb{R}_{+} \rightarrow\left[0, \bar{F}_{i}\right], \bar{F}_{i} \geq$ 0 .

The entries of $K$ relate the reactions as follows:

$k_{i j}=c_{i j} \quad>0$ if, and only if, $i \in R_{j} \backslash\left(L_{j} \cap R_{j}\right)$, $k_{i j}=0$ if, and only if, $i \in R_{j} \cap L_{j}$

$$
\text { or } i \notin R_{j} \cup L_{j} \text {, }
$$

$k_{i j}=-c_{i j}<0$ if, and only if, $i \in L_{j} \backslash\left(L_{j} \cap R_{j}\right)$.

Notice that all of the entries of $K$ are determined by (3) since $\{1, \ldots, N\}$ is the disjoint union of the sets $R_{j} \backslash\left(L_{j} \cap R_{j}\right), L_{j} \backslash\left(L_{j} \cap R_{j}\right), R_{j} \cap L_{j}$ and $\{1, \ldots, N\} \backslash\left(R_{j} \cup L_{j}\right)$.

Notice also that $\underline{D}>0$ means that we consider a continous stirred tank reactor.

Example 1. The reaction scheme

$$
\begin{aligned}
3 \xi_{1} & \stackrel{\varphi_{1}}{\longrightarrow} 2 \xi_{2}+\xi_{3} \\
2 \xi_{4}+3 \xi_{2} & \stackrel{\varphi_{2}}{\longrightarrow} 4 \xi_{1}+\xi_{5} \\
\xi_{1}+\xi_{2} & \stackrel{\varphi_{3}}{\longrightarrow} 2 \xi_{4} \\
3 \xi_{6} & \stackrel{\varphi_{4}}{\longrightarrow} 1 \xi_{2}+2 \xi_{7}
\end{aligned}
$$

has the stoichiometric matrix

$$
K=\left[K_{1}, K_{2}, K_{3}, K_{4}\right]=\left[\begin{array}{cccc}
-3 & 4 & -1 & 0 \\
2 & -3 & -1 & 1 \\
1 & 0 & 0 & 0 \\
0 & -2 & 2 & 0 \\
0 & 1 & 0 & 0 \\
0 & 0 & 0 & -3 \\
0 & 0 & 0 & 2
\end{array}\right]
$$

We stress that, although the matrix $K$ is called stoichiometric matrix, we do not require that it represents an exact stoichiometric relationship between the components, it represents a qualitative relationship. Components that do not play an important role in the process, such as by-products of a reaction or substrates which are not limiting, have been omitted. The advantage of this description is that the model might be of much smaller dimension. This approach is quite common; see for instance Bastin and Dochain (1990). However, the reaction scheme may not follow the law of conservation of mass. We therefore have to prove that (2) still exhibits practically relevant features, such as existence and uniqueness, positivity and boundedness of the solution.

Before this will be done in Section 2, we compare the basic assumption we will make on (2) with models which do assume conservation of mass. The most prominent reference on chemical reacting systems is Gavalas (1968). See in particular Section 1.1, where he introduces systems which can be described by an ordinary differential equation of the form (2). Although Gavalas does not explicitly say so (see Section 1.1 and also the sentence below equation (1.8.11)), the Principle of Mass Conservation implies the existence of a positive vector

$\gamma \in\left(\mathbb{R}_{+}^{*}\right)^{N}$ so that $\gamma^{T} K_{j}=0$ for all $j=1, \ldots, M .(4)$

(3) Intuitively, this property means that the concentration remains bounded and is in fact crucial in proving it.

Instead of conservation of mass, we introduce the new notion of non-cyclic processes. Cyclic means roughly that the process contains a reaction loop, i.e. there exists a subset of reactions $S$, so that every substrate $\xi_{i}$ involved in a reaction $j \in S$ is also an autocatalyst or a product of one of the reactions $j \in S$. One of the crucial consequences of non-cyclic processes is that there exists a positive vector

$\gamma \in\left(\mathbb{R}_{+}^{*}\right)^{N}$ so that $\gamma^{T} K_{j} \leq 0$ for all $j=1, \ldots, M .(5)$

Note that the condition (5) is only slightly less restrictive than (4). However it is sufficient to guarantee boundedness of the trajectories.

This note is organized as follows: In Section 2 we prove, provided (5) and an additional assumption on the growth rates holds, boundedness of the solution of (2) and invariance of the positive orthant. In Section 3 we intoduce the new concept of noncyclic processes, prove that it implies (5), present an algorithm which decides in finitely many steps whether a process is non-cyclic or not and state a characterization of it in terms of a normal form of the stoichiometric matrix $K$. In Section 4 we show how to control an external substrate of (2) into a prespecified neighbourhood of a constant reference signal by using the feedrate as control variable. This controller is an adaptive high-gain 
controller which copes with input constraints and noise corrupting the output.

\section{DYNAMICS OF THE MODEL}

In this section we show that the model (2), although is does not obey the conservation of mass, exhibits such properties as boundedness and invariance of the positive orthant. To this end, we have to be more specific with the sets $L_{j}$ and $R_{j}$ and introduce:

$$
\operatorname{Cat}_{j}:=L_{j} \cap R_{j},
$$

i.e. the set of the indices of those components, called catalysts, which are involved in the $j$-th reaction, but maintained by the reaction;

$$
S u b_{j}:=L_{j} \backslash\left(L_{j} \cap R_{j}\right) \neq \emptyset,
$$

i.e. the set of those components, called substrates, that are consumed by the $j$-th reaction;

$$
\operatorname{Prod}_{j} \subseteq R_{j} \backslash\left(L_{j} \cap R_{j}\right) \neq \emptyset
$$

i.e. the set of the indices of those components, called products, that are produced by the $j$-th reaction;

$$
A u t_{j}:=R_{j} \backslash\left(\left(L_{j} \cap R_{j}\right) \cup \operatorname{Prod}_{j}\right),
$$

i.e. the set of the indices of those components, called autocatalysts, that are accumulated by the $j$-th reaction;

$$
\text { Rest }_{j}:=\{1, \ldots, N\} \backslash\left(L_{j} \cup R_{j}\right),
$$

i.e. the set of the process components that are not involved in the $j$-th reaction.

Notice that, for all $j=1, \ldots, M$,

$$
A u t_{j} \cup \operatorname{Prod}_{j}=R_{j} \backslash\left(R_{j} \cap L_{j}\right)
$$

and $\{1, \ldots, N\}$ can be represented as the disjoint union:

$$
\{1, \ldots, N\}=C a t_{j} \dot{\cup} S u b_{j} \dot{\cup} A u t_{j} \dot{\cup} \operatorname{Prod}_{j} \dot{\cup} \operatorname{Rest}_{j} .
$$

A crucial assumption on the reaction rates is, that they are of the form, for all $j=1, \ldots, M$,

$(\mathbf{A 1})\left\{\begin{array}{l}\xi \mapsto \varphi_{j}(\xi)=\alpha_{j}(\xi) \cdot\left(\prod_{k \in A u t_{j} \cup L_{j}} \xi_{k}\right), \\ \text { with continuous growth rate functions } \\ \alpha_{j}(\cdot): \mathbb{R}_{+}^{N} \longrightarrow\left(\underline{\alpha}_{j}, \bar{\alpha}_{j}\right], \quad 0 \leq \underline{\alpha}_{j} \leq \bar{\alpha}_{j} .\end{array}\right.$

For a comprehensive list of reaction rates see for instance the Appendix in Bastin and Dochain (1990). The reaction rate $\varphi$ is often assumed to be proportional to the microbial growth rate $\mu$. The most prominent ones are the models of Monod or Haldane.
Proposition 2. Suppose (2) satisfies (A1). Then for each initial condition $\xi(0) \in\left(\mathbb{R}_{+}^{*}\right)^{N}$ there exists a unique solution $\xi(\cdot):[0, \infty) \longrightarrow\left(\mathbb{R}_{+}^{*}\right)^{N}$, that means invariance of the positive orthant. If, in addition, (5) holds, then this solution is bounded.

The following characterizations of the catalysts, substrates and products will be used in the sequel:

$$
\left.\begin{array}{rl}
\mathrm{Cat}_{j} & =\left\{i \in L_{j} \cup R_{j} \mid k_{i j}=0\right\} \\
S u b_{j} & =\left\{i \in\{1, \ldots, N\} \mid k_{i j}<0\right\} \\
\operatorname{Prod}_{j} \cup A u t_{j} & =\left\{i \in\{1, \ldots, N\} \mid k_{i j}>0\right\}
\end{array}\right\}
$$

Proof of Proposition 2: Existence and uniqueness of a solution $\xi(\cdot):[0, \omega) \longrightarrow \mathbb{R}^{N}$ of $(2)$ on a maximal interval of existence $[0, \omega)$, for some $\omega \in(0, \infty]$, follows from the theory of ordinary differential equations. We have to prove boundedness (which implies $\omega=\infty$ ) and invariance of the positive orthant.

Seeking a contradiction to the invariance of the positive orthant under the flow, suppose there exist some $i \in\{1, \ldots, N\}$ and $t_{0} \in[0, \omega)$ such that

$\xi_{i}\left(t_{0}\right)=0$ and $\xi_{k}(t)>0 \forall t<t_{0}, k=1, \ldots, N$.

The $i$-th coordinate of (2) satisfies, for all $t \in$ $\left[0, t_{0}\right]$,

$\dot{\xi}_{i}(t)=\sum_{j=1}^{M} k_{i j} \varphi_{j}(\xi(t))-D(t) \xi_{i}(t)-q_{i} \xi_{i}(t)+F_{i}(t)$.

Let the set of numbers of the reactions that involve $\xi_{i}$ as a substrate be defined as

$$
R_{S u b_{i}}:=\left\{j \in\{1, \ldots, M\} \mid k_{i j}<0\right\} .
$$

By (6) we know that for $j \notin R_{S u b_{i}}, k_{i j} \geq 0$, and since $F_{i}(t) \geq 0$ for all $t \geq 0$ it follows that

$\dot{\xi}_{i}(t) \geq-\sum_{j \in R_{S \mathbf{u} b_{i}}}\left|k_{i j}\right| \varphi_{j}(\xi(t))-D(t) \cdot \xi_{i}(t)-q_{i} \cdot \xi_{i}(t)$

Therefore by using the notation

$$
\begin{aligned}
& \psi_{i}(t):= \\
& -\sum_{j \in R_{S u b_{i}}}\left|k_{i j}\right| \alpha_{j}(\xi(t))\left(\prod_{k \in\left(A u t_{j} \cup L_{j}\right) \backslash\{i\}} \xi_{k}(t)\right)-D(t)-q_{i}
\end{aligned}
$$

and (A1), we can prove that

$$
\dot{\xi}_{i}(t) \geq \psi_{i}(t) \cdot \xi_{i}(t),
$$

and integration yields

$$
\xi_{i}\left(t_{0}\right) \geq e^{\int_{0}^{t_{0}} \psi_{i}(\tau) d \tau} \xi_{i}(0)
$$


Since $\xi_{i}(0)>0$, this contradicts the assumption $\xi_{i}\left(t_{0}\right)=0$ in $(7)$. Therefore a component cannot leave the positive orthant and the state variables stay positive on their maximal interval of existence.

It remains to prove boundedness. Let $\gamma$ be given as in (5) and define

$$
\Phi(t):=\gamma^{T} \xi(t)
$$

Then multiplication of (2) from the left with $\gamma^{T}$ yields, for almost all $t \in[0, \omega)$,

$$
\begin{gathered}
\dot{\Phi}(t)=\sum_{j=1}^{M}\left\langle\gamma, K_{j}\right\rangle \varphi_{j}(\xi(t))-D(t) \Phi(t) \\
-\sum_{j=1}^{N} \gamma_{j} q_{j} \xi_{j}(t)+\langle\gamma, F(t)\rangle,
\end{gathered}
$$

and hence, by (5) and positivity of $\xi(t)$,

$$
\dot{\Phi}(t) \leq-D(t) \cdot \Phi(t)+\langle\gamma, F(t)\rangle \text {. }
$$

Since $\underline{D}>0$, an application of the Variations-ofConstants formula yields, for all $t \geq 0$,

$$
|\Phi(t)| \leq e^{-\underline{D} t}\left|\Phi\left(t_{0}\right)\right|+\int_{0}^{t} e^{-\underline{D}(t-s)} \gamma^{T} F(s) d s,
$$

and boundedness of $\Phi(\cdot)$ is a consequence of boundedness of $F(\cdot)$. This completes the proof.

\section{NON-CYCLIC PROCESSES}

In this section we introduce the concept of noncyclic processes. This is satisfied by numerous biochemical models of processes, also when they do not obey conservation of mass. Most importantly, if a process (2) is non-cyclic, then (5) is satisfied and therefore Proposition 2 guarantees existence and boundedness of the solution in the positive orthant.

Cyclicity of the general dynamical model (2) means that the process contains a reaction loop, i.e. there exists a subset of reactions $S$, so that every substrate $\xi_{i}$ involved in a reaction $j \in S$ is also an autocatalyst or a product of one of the reactions $j \in S$. Or in other words, (2) is noncyclic respectively does not contain a reaction loop if, and only if, for each subset $S$ there exists a substrate $\xi_{i}$ of some reaction $j_{0} \in S$ such that $\xi_{i}$ is neither an autocatalyst nor a product of one of the reactions $j \in S$. This concept is more formally defined as follows.

Definition 3. The biotechnological process (2) is said to be cyclic if, and only if, there exists a nonempty subset $S \subseteq\{1, \ldots, M\}$, such that

$$
\bigcup_{j \in S} S u b_{j} \subseteq \bigcup_{j \in S}\left(A u t_{j} \cup \operatorname{Prod}_{j}\right) \text {. }
$$

Theorem 4. Non-cyclicity of (2) is sufficient but not necessary for (5).

The proof of Theorem 4 is too long that it can be presented here in full detail; see Ilchmann and Weirig (1997). It is mainly based on the following algorithm which decides in finitely many iterations whether (2) is cyclic or not.

Algorithm 1. Consider the biotechnological process (2) with specified $M, N, S u b_{j}, A_{u} t_{j}, \operatorname{Prod}_{j}$ for $j=1, \ldots, M$.

$$
\begin{aligned}
\text { SET } S_{1}:=\{1, \ldots, M\} \\
\mathcal{N}:=\{1, \ldots, N\} \\
\text { FOR } l=1, \ldots, M
\end{aligned}
$$

DO IF $\quad(l>1)$ and there exists some $j_{l} \in S_{l}$ such that $\left(i_{l-1} \in S u b_{j_{l}}\right)$ SET $i_{l}:=i_{l-1}$; GOTO 10 ;

ELSE IF there exists $\left(i_{l}, j_{l}\right) \in \mathcal{N} \times S_{l}$ such that $i_{l} \in S u b_{j_{l}}$ and $i_{l} \notin \bigcup_{j \in S_{l}}\left(A u t_{j} \cup \operatorname{Prod}_{j}\right)$ GOTO 10 ;

ELSE PRINT "cyclic";

10. $S_{l+1}:=S_{l} \backslash\left\{j_{l}\right\}$;

END

END "non-cyclic"
PRINT
END.

The following example shows one 'direction' of Theorem 4, namely that non-cyclic is not necessary for (5).

Example 5. Considering again Example 1. For $\gamma^{T}:=(1, \ldots, 1)$ and $j=1,2,3,4$, we have $\gamma^{T} K_{j}=0$ and hence (5) is satisfied.

However, the process is cyclic, since for the subset $S:=\{1,2,3\}$ we have

$$
\bigcup_{j \in S} S u b_{j}=\{1,2,4\} \subseteq \bigcup_{j \in S}\left(A u t_{j} \cup \operatorname{Prod}_{j}\right)=\{1, \ldots, 5\} \text {. }
$$

Remark 6. Having established Algorithm 1 one can formulate, by using (6), the algorithm also in terms of the stoichiometric matrix $K$ : In IF one has to replace ' $\left(i_{l-1} \in S u b_{j_{l}}\right)$ ' by ' $k_{i_{l-1} j_{l}}<0$ ', and in ELSE IF one replaces ' $i_{l} \in S u b_{j_{l}}$ and $i_{l} \notin$ $\bigcup\left(A u t_{j} \cup \operatorname{Prod}_{j}\right)$ ' by ' $k_{i_{l} j_{l}}<0$ and $k_{i_{l} j} \leq 0$ for $j \in S_{l}$

all $j \in S_{l}$ '. (For a proof see Weirig (1998).)

Non-cyclic processes can be decomposed into equivalent classes and the following theorem describes the representatives. 
Theorem 7. The biotechnological process (2) is non-cyclic if, and only if, there exist permutation matrices $L \in \mathbb{R}^{N \times N}$ and $R \in \mathbb{R}^{M \times M}$ such that

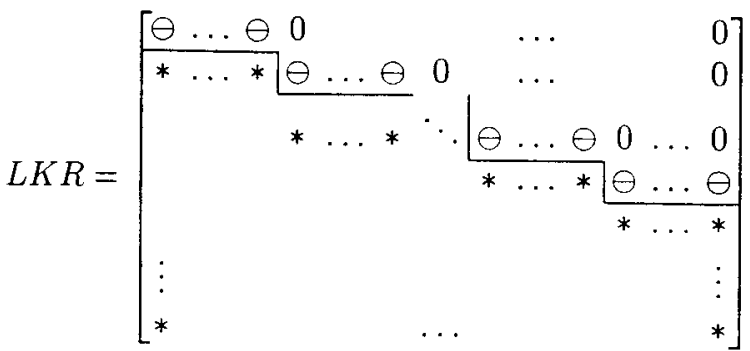

where $\ominus$ denotes negative coefficients and $*$ are some real coefficients.

For a proof see Weirig (1998). The above echelon form is not unique.

\section{AN EXAMPLE FOR $\lambda$-TRACKING IN THE PRESENCE OF INPUT CONSTRAINTS}

In this section we design a single adaptive controller which achieves tracking of constant substrate concentration in the presence of input constraints for a whole class of systems, the exact systems parameter need not to be known. Here the control objective is to track the reference signal asymptotically only within a prespecified neighbourhood; the tracking error is supposed to become as small as one likes but not necessarily zero. The controller is a simple time-varying error feedback where the gain is tuned adaptively by the size of the error signal only. This approach is in the spirit of Ilchmann and Ryan (1994). It does not invoke any identification mechanism, internal model or probing signals. Moreover the control structure can cope with input saturations provided a certain feasibility assumption is satisfied.

We are currently working on a general concept. However, here we will only present this approach for a simple example which captures the essence.

Suppose there exists an external substrate $\xi_{l}$ of the general reactor model (2), i.e.

$$
l \in \bigcup_{j=1}^{M} S u b_{j} \backslash \bigcup_{j=1}^{M}\left(A u t_{j} \cup \operatorname{Prod}_{j}\right) .
$$

This substrate is viewed as the output variable to be controlled. The control objective is to drive the concentration $\xi_{l}$ in a prespecified $\lambda$-strip around a constant reference signal $\xi_{\text {ref }}$. The control variable is the $l$-th feedrate $F_{l}(\cdot)$.

A further assumption to be made is the existence of upper bounds of the reaction rates, i.e. (see (8)

$$
\text { (A2) }\left\{\begin{array}{l}
\text { for each } j \in R_{S u b_{l}} \text { there exists some } \\
\bar{\varphi}_{j} \geq \sup \left\{\left|\varphi_{j}(\xi)\right| \mid \xi \in\left(\mathbb{R}_{+}^{*}\right)^{N}\right\} .
\end{array}\right.
$$

The knowledge of upper bounds of the reaction rates can be guaranteed if the reaction rates do not depend on the concentration of the autocatalysts. This is for instance the case when there are no autocatalysts involved in the reactions $j \in R_{S u b_{t}}$. Then the models of Monod or Haldane give upper bounds.

Some other examples of bounded reaction rates, even depending on the concentration of the autocatalysts, are given in Appendix 1 in Bastin and Dochain (1990).

In order to regulate the error between the external substrate and the constant reference signal, i.e.

$$
e(t):=\xi_{l}(t)-\xi_{\text {ref }},
$$

into the $\lambda$-strip around 0 , we apply the following feedback law

$$
u(t)=\operatorname{sat}_{\left[0, \bar{F}_{l}\right]}(-k(t) e(t)+\delta(t)),
$$

where, for $a<b$, we define

$$
\operatorname{sat}_{[a, b]}(\eta):=\left\{\begin{array}{l}
a, \eta<a \\
\eta, \eta \in[a, b] \\
b, \eta>b .
\end{array}\right.
$$

The time-varying gain $k(t)$ is tuned adaptively, for some prespecified $\gamma, \lambda>0$, by

$$
\dot{k}(t):=\left\{\begin{array}{cl}
\gamma(|e(t)|-\lambda)^{2}, & |e(t)| \geq \lambda \\
0, & |e(t)|<\lambda
\end{array}\right.
$$

Note that the gain adptation $t \mapsto k(t)$ is monotonically non-decreasing. The idea is that once the gain is sufficiently large, the error is forced into the $\lambda$-strip and the gain adaptation is switched off, that means $k(t)$ stays constant.

In the following theorem we show that $\lambda$-tracking is possible in the presence of input saturations, provided the input constraints satisfy a feasibility assumption in terms of the system and control data. This is the price to be paid if input constraints are applied.

Theorem 8. Suppose the general reactor model (2) satisfies (A1), (A2), (5) and $\delta(\cdot):[0, \infty) \longrightarrow$ $[0, \bar{\delta}]$ is a differentiable function with bounded derivative and $\bar{\delta} \geq 0$.

If $\xi_{\text {ref }} \in(\lambda, \infty)$ is a constant reference signal which satisfies the feasibility condition

$$
\bar{F}_{l} \geq \sum_{j=1}^{M}\left|k_{l j}\right| \bar{\varphi}_{j}+q_{l} \cdot\left(\xi_{\text {ref }}-\lambda\right)+\bar{D} \cdot \xi_{\text {ref }},
$$

then, for arbitrary but prespecified $\gamma, \lambda>0$, the $\lambda$-tracker (10), (11) applied to the reactor process (2) yields, for every initial condition $\xi(0) \epsilon$ $\left(\mathbb{R}_{+}^{*}\right)^{N}, k(0) \in \mathbb{R}$, a unique solution

$$
\xi(\cdot):[0, \infty) \longrightarrow\left(\mathbb{R}_{+}^{*}\right)^{N}
$$


of the closed-loop system (2), (10), (11). This solution satisfies

- $\xi(\cdot) \in L_{\infty}(0, \infty)$,

- $\lim _{t \rightarrow \infty} k(t)=k_{\infty} \in \mathbb{R}_{+}$,

- $\lim _{t \rightarrow \infty} \operatorname{dist}\left(\xi_{l}(t)-\xi_{\text {ref }},[-\lambda, \lambda]\right)=0$.

Before proving this theorem, we will interprete the design parameters. The size of the dead-zone $\lambda>$ 0 should be larger than the measurement accuracy plus an additional width for measurement noise, if an upper bound of the latter is known in advance. $\gamma>0$ adjusts the speed of adaptation and a sensible choice lies in the order of magnitude of the inverse of the "dominate time constant" of the plant. The signal $\delta(\cdot)$ could be viewed as a disturbance but we rather use it as an appropriate control input which might be known to the designer from experience.

Proof of Theorem 8: Existence, uniqueness and boundedness of the solution $\xi(\cdot)$ follows from Proposition 2. $k(\cdot)$ cannot have a finite escape time since it is the integral of a bounded function.

In order to show boundedness of $k(\cdot)$ we suppose, by seeking a contradiction,

$$
\lim _{t \rightarrow \infty} k(t)=\infty
$$

and proceed in several steps.

Choose $\bar{t}>0$ such that

$$
-k(\bar{t}) \cdot \lambda+\bar{\delta}<0 \text { and } k(\bar{t}) \cdot \lambda>\bar{F}_{l} .
$$

STEP 1: We show that if $\xi_{l}(\bar{t}) \geq \xi_{\text {ref }}+\lambda$, then there exists $t_{1}>\bar{t}$ such that $\xi_{l}\left(t_{1}\right)<\xi_{\text {ref }}+\lambda$. If we assume that

$$
\xi_{l}(t) \geq \xi_{\text {ref }}+\lambda \text { for all } t \geq \bar{t},
$$

then similar to the proof of Proposition 2 we can prove

$$
\xi_{l}(t) \leq e^{-\underline{D} \cdot\left(t-t_{0}\right)} \xi_{l}\left(t_{0}\right)
$$

which contradicts (14).

STEP 2: We show that if $\xi_{l}(\bar{t}) \leq \xi_{\text {ref }}-\lambda$, then there exists some $t_{1}>\bar{t}$ such that $\xi_{l}\left(t_{1}\right)>\xi_{\text {ref }}-\lambda$. This is proved similarly as Step 1. Assuming, by contradiction, that

$$
\xi_{l}(t) \leq \xi_{\mathrm{ref}}-\lambda \text { for all } t \geq \bar{t},
$$

we end up with

$$
\dot{\xi}_{l}(t) \geq \underline{D} \cdot \lambda \text { for all } t \geq \bar{t},
$$

which condradicts the assumption.

STEP 3: By Step 1 and 2 we may choose some $t_{1} \geq \bar{t}$ such that

$$
\xi_{l}\left(t_{1}\right) \in\left(\xi_{\text {ref }}-\lambda, \xi_{\text {ref }}+\lambda\right) .
$$

We now show that

$$
\xi_{l}(t) \in(-\lambda, \lambda) \text { for all } t \geq t_{1} .
$$

Suppose there exists some $t_{2}>t_{1}$ such that

$$
\xi_{l}\left(t_{2}\right)=\xi_{\text {ref }}+\lambda
$$

then, analogously to Step 1 , we may conclude that $\dot{\xi}_{l}\left(t_{2}\right)<0$, which contradicts the assumption. Thus we cannot hit the upper bound of the $\lambda$ strip. In a similar manner one shows that it is impossible to hit the lower bound.

STEP 4: Step 3 contradicts unboundedness of $k(\cdot)$ and the second statement of the theorem is established. To prove the last statement, differentiate the Lyapunov function candidate

$$
V(\cdot): \mathbb{R}_{+} \times \mathbb{R} \times \mathbb{R}_{+} \longrightarrow \mathbb{R}, \quad(t, e, k) \mapsto-k
$$

along the solution of the closed-loop system (2), (10), (11). Note the $V$ is sign indefinite, but boundedness of the trajectories is already established. Since $d / d t V(t, e(t), k(t))=-\dot{k}(t)$, it follows from LaSalle's Invariance Principle that the $\omega$-limit set of the solutions is included in the set $\left\{(t, e, k) \in \mathbb{R}_{+} \times \mathbb{R} \times \mathbb{R}_{+} \mid \dot{k}=0\right\}$, which is the set $\{e \in \mathbb{R} \mid\|e\| \leq \lambda\}$. Hence

$\lim _{t \rightarrow \infty} \operatorname{dist}(e(t),[-\lambda, \lambda])=0$, which completes the proof.

\section{References}

Bastin, G. and D. Dochain (1990). On-line Estimation and Adaptive Control of Bioreactors. Elsevier Science Pubs. B.V., Amsterdam et al.

Gavalas, G.R. (1968). Nonlinear Differential Equations of Chemically Reacting Systems. SpringerVerlag, Berlin et al.

Ilchmann, A. and E.P. Ryan (1994). Universal $\lambda$ tracking for nonlinearly perturbed systems in the presence of noise. Automatica, 30, 337-346.

Ilchmann, A. and M.-F. Weirig (1997). Modelling of general biotechnological processes. Dept. of Maths., Report M97/12, University of Exeter.

Weirig, M.-F. (1998). Modelling and adaptive control of biotechnological processes. $\mathrm{PhD}$ thesis, submitted to the Department of Mathematics, Technical University Munich. 